\title{
Effect of Molecular Weight Dependence of the $\chi$-Parameter on Phase Equilibrium of Multicomponent Polymer-Single Solvent System: Comparison of Theory with Experiment
}

\author{
Kenji KAMIDE and Yukio MIYAZAKI \\ Textile Research Laboratory, Asahi Chemical Industry Company, Ltd., \\ Hacchonawate Takatsuki, Osaka 569, Japan.
}

(Received June 30, 1980)

\begin{abstract}
A generalized theory has been established for describing the phase equilibrium of multicomponent polymer-single solvent system, by introducing the concept of molecular weightand concentration-dependence of the polymer-solvent thermodynamical interaction parameter $\chi$ to the previous theory. In this theory $\chi$ is expressed by

$$
\chi=\chi_{00}(1+k / X)\left(1+p \cdot v_{\mathrm{p}}\right)
$$

where, $\chi_{00}$ is a constant independent of both polymer molecular weight and concentration, $X$, the molar volume ratio of $X$-mer to the solvent, $v_{\mathrm{p}}$, the polymer volume fraction, $k$ and $p$, the molecular weight- and concentration-dependence coefficients, respectively. The mathematical phase separation and fractionation were carried out and the effect of $k$ - and $p$-parameters on the phase separation and fractionation characteristics were studied in detail. The positive molecular weight dependence of $\chi$ paramerer $(k>0)$ indices a large polydispersity of the polymers in the polymer-rich and lean-phases, particularly where $k>3$. In the small $\rho_{\mathbf{p}}\left(\rho_{\mathbf{p}}=\right.$ the relative weight fraction of the polymer in a polymer-rich phase to the total polymer dissolved) region, the ratio of the weight- to number-average $X, X_{w} / X_{n}$ of the polymer remaining in a polymer-rich phase is remarkably influenced by both $p$ and $k$. In a large $\rho_{\mathrm{p}}$ region, the polydispersity of the polymer in a polymer-lean phase is predominantly controlled by $k$. For polystyrene-cyclohexane and polystyrenemethylcyclohexane systems, $k$, estimated by three methods, was found to lie between 0 and 10 . The $k$ values for these systems decrease with increasing temperature.
\end{abstract}
KEY WORDS $\chi$-Parameter / Phase Equilibrium / Multicomponent Polymer /
Single Solvent System / Computer Simulation / Molecular Weight
Fractionation /

For the past several years, calculations based on a very rigorous theory of phase equilibrium have been extensively carried out by Kamide and his coworkers for multicomponent polymer-single solvent systems. ${ }^{1}$ They took into account the concentration dependence of the polymer-solvent interaction parameter $\chi$, which has also been studied by membrane osmometry, spinodal and critical miscibility. Kamide et al. ${ }^{2,3}$ have demonstrated on polystyrene-cyclohexane (PS-CH) and polystyrene-methylcyclohexane (PS-MCH) systems that the phase separation characteristics including volume ratio of a polymer-lean phase and a polymer-rich phase $R$ coincide well with the results of computer simulation experiments when the latter were performed by using, for the concentration dependence coefficient, $p$ of the $\chi$ parameter, ( $p \equiv\left\{\left(\chi / \chi_{0}\right)-1\right\} / v_{\mathrm{p}} \cdot \chi_{0}$ is a parameter depending on temperature and independent of concentration, and $v_{\mathrm{p}}$ is the polymer volume fraction.). This value has been determined by other methods, such as membrane osmometry. ${ }^{4}$ The partition coefficient $\sigma$ determined experimentally for the PS-CH and PS$\mathrm{MCH}$ systems showed a small but significant tendency to decrease with increasing molecular weight, $M .^{2}$ For example, among 16 phaseseparation experiments for PS- $\mathrm{CH}$ and PS-MCH systems, the plots of $\sigma$ (eq 12) against the reciprocal 
molar ratio of $X$-mer to the solvent for 14 experiments exhibited positive slopes, and the slopes of the plots for the rest were positive in the region of small $X^{-1}$ and changed to negative in the region of large $X^{-1}$. It should be noted that the latter two experiments involved a comparatively large experimental error due to small $\sigma$ values obtained. We tried to attribute the molecular weight dependence of $\sigma$ to the molecular weight dependence of the $\chi$ parameter. $^{2}$ Koningsveld and Kleintjens ${ }^{5}$ and Kennedy et al. ${ }^{6}$ have pointed out that the molecular weight dependence of the $\chi$-parameter influences significantly spinodal and critical miscibility. Unfortunately, a more sophisticated theory for phase separation of solutions in which the molecular weight and concentration dependences of the $\chi$ parameter are adequately considered, is not as yet established.

In this article, we carry out a theoretical calculation of the phase separation and successive fractionation on the quasibinary system, with the molecular weight- and concentration-dependences of the $\chi$-parameter taken into account. We did this so as to get a better understanding of effect of molecular weight-dependence of the $\chi$-parameter on phase-separation phenomena. A comparison is made with the experimental data in the previous paper. $^{2}$

\section{THEORETICAL BACKGROUND}

The polymer-solvent thermodynamic interaction parameter $\chi$ can be expressed approximately by

$$
\chi=\chi_{00}\left\{1+\left(\chi_{01} / \chi_{00}\right) / X\right\}\left(1+p \cdot v_{\mathrm{p}}\right)
$$

where $\chi_{00}$ and $\chi_{01}$ are constants independent of both polymer molecular weight (in this case, molar volume ratio of $X$-mer to solvent, $X$ ) and polymer concentration (polymer volume fraction $v_{\mathrm{p}}$ ). The ratio $\chi_{01} / \chi_{00}$ is a parameter representing the molecular weight dependence and $p$ is a concentration dependence parameter. Obviously, $\chi_{00}$ and $\chi_{01}$ are temperature-dependent.

According to the refined solution lattice theory, the parameters $\chi_{00}$ and $\chi_{01}$ are given $b^{2}$

$$
\begin{gathered}
\chi_{00}=1 / Z+B^{\prime} /\left(k^{\prime} \cdot T\right) \\
\chi_{01}=-2 / Z
\end{gathered}
$$

where $Z$ is the coordination number of a lattice site, $k^{\prime}$, the Boltzmann constant, $T$, absolute temperature, and $B^{\prime}$, the proportionality constant between the partial molar heat of dilution, $\Delta H_{0}$ and $v_{\mathrm{p}}{ }^{2}$, i.e.,

$$
B^{\prime}=\lim _{v_{\mathrm{p}} \rightarrow 0} \Delta H_{0} / v_{\mathrm{p}}^{2} .
$$

Equations 2 and 3 were derived assuming the van Laar-type equation for the heat of mixing, which is not, in the strict sense, applicable to the polymer solution. Also, the parameter $B^{\prime}$ is molecular weight dependent. Therefore, the parameters $\chi_{00}$ and $\chi_{01}$ should be at present regarded as empirical parameters. Very recently, Fujiwara ${ }^{7}$ derived a theory describing the entropy and enthalpy terms $\chi_{\mathrm{s}}$ and $\chi_{\mathrm{h}}$ in the $\chi$-parameter $\left(\chi=\chi_{s}+\chi_{h}\right)$ through out the entire concentration region, by introducing the concept of a hypothetical sphere domain with definite dimensions occupied by a polymeric chain in solution. In his theory, the ratio $\chi_{01} / \chi_{00}$ and $p$ parameter in eq 1 are given in the terms of the concentration and molecular weight dependence of the hypothetical sphere. Consequently, at least the molecular weight dependence of the enthalpy term of the $\chi_{0}$ parameter (designated as $\chi_{0, \mathrm{~h}}$ ) can be qualitatively explained.

Substitution of eq 1 into the well-known FloryHuggins equations for the partial molar free energy of dilution of the solvent, $\Delta \mu_{0}$, and that of $X$-mer, $\Delta \mu_{x}$, gives,

$$
\begin{gathered}
\Delta \mu_{0}=\bar{R} T\left[\ln \left(1-v_{\mathrm{p}}\right)+\left(1-1 / X_{n}\right) v_{\mathrm{p}}\right. \\
\left.+\chi_{00}\left(1+k / X_{n}\right)\left(1+p \cdot v_{\mathrm{p}}\right) v_{\mathrm{p}}{ }^{2}\right]
\end{gathered}
$$

and,

$$
\begin{aligned}
& \Delta \mu_{x}=\bar{R} \cdot T\left[\ln v_{x}-(X-1)+X\left(1-1 / X_{n}\right) v_{\mathrm{p}}\right. \\
& \quad+\chi_{00} X\left(1+\mathrm{k} / X_{n}\right)\left\{\left(1-v_{\mathrm{p}}\right)^{2}+p\left(0.5-1.5 \cdot v_{\mathrm{p}}{ }^{2}+v_{\mathrm{p}}{ }^{3}\right)\right\} \\
& \left.+\chi_{00} k X\left(1 / X-1 / X_{n}\right)\left\{\left(1-v_{\mathrm{p}}\right)+0.5 \cdot p\left(1-v_{\mathrm{p}}{ }^{2}\right)\right\}\right](5)
\end{aligned}
$$

where,

$$
k=\chi_{01} / \chi_{00}
$$

$X_{n}$ is the number-average molar volume ratio of polymer to solvent, $v_{x}$, the volume fraction of $X$ mer, $\bar{R}$, the gas constant, $T$, the absolute temperature. Equation A 2 in the previous paper ${ }^{2}$ should be corrected to eq 5 .

When two liquid phases are in thermodynamic equilibrium, the following conditions should be satisfied, 


$$
\begin{aligned}
\Delta \mu_{0(1)} & =\Delta \mu_{0(2)} \\
\Delta \mu_{x(1)}= & \Delta \mu_{x(2)} \\
& \text { for } \quad X=1,2, \cdots
\end{aligned}
$$

phase and polymer-rich phase, respectively.

From eq $4-8$ it follows that the partition

where indices (1) and (2) represent the polymer-lean is represented as

$$
\begin{aligned}
\sigma=\left(v_{\mathrm{p}(2)}-v_{\mathrm{p}(1)}\right) & +\left(v_{\mathrm{p}(2)} / X_{n(2)}-v_{\mathrm{p}(1)} / X_{n(1)}\right) \\
& +\chi_{00}\left[\left(1+k / X_{n(1)}\right)\left\{\left(1-v_{\mathrm{p}(1)}\right)^{2}+p\left(0.5-1.5 v_{\mathrm{p}(1)}^{2}+v_{\mathrm{p}(1)}^{3}\right)\right\}\right. \\
& -\left(1+1 / X_{n(2)}\right)\left\{\left(1-v_{\mathrm{p}(2)}\right)^{2}+p\left(0.5-1.5 v_{\mathbf{p}(2)}^{2}+v_{\mathbf{p}(2)}^{3}\right\}\right. \\
& +k\left(1 / X-1 / X_{n(1)}\right)\left\{\left(1-v_{\mathbf{p}(1)}\right)+0.5 p\left(1-v_{\mathbf{p}(1)}^{2}\right)\right\} \\
& \left.-k\left(1 / X-1 / X_{n(2)}\right)\left\{\left(1-v_{\mathbf{p}(2)}\right)+0.5 p\left(1-v_{\mathbf{p}(2)}^{2}\right)\right\}\right]
\end{aligned}
$$

$$
\sigma=1 / X \ln \left(v_{x(2)} / v_{x(1)}\right)
$$

$$
\chi_{00} \text { in eq } 10 \text { can be rewritten as follows }
$$

$$
\chi_{00}=\frac{\ln \frac{\left(1-v_{\mathrm{p}(1)}\right)}{\left(1-v_{\mathrm{p}(2)}\right)}+\left(v_{\mathrm{p}(1)}-v_{\mathrm{p}(2)}\right)+\left(\frac{v_{\mathrm{p}(2)}}{X_{n(2)}}-\frac{v_{\mathbf{p}(1)}}{X_{n(1)}}\right)}{\left(v_{\mathbf{p}(2)}^{2}-v_{\mathrm{p}(1)}^{2}\right)+p\left(v_{\mathbf{p}(2)}^{3}-v_{\mathrm{p}(1)}^{3}\right)+k\left[\left(\frac{v_{\mathrm{p}(2)}^{2}}{X_{n(2)}}-\frac{v_{\mathrm{p}(1)}^{2}}{X_{n(1)}}\right)+p\left(\frac{v_{\mathrm{p}(2)}^{3}}{X_{n(2)}}-\frac{v_{\mathrm{p}(1)}^{3}}{X_{n(1)}}\right)\right]}
$$

Then, substituting eq 11 into eq 10 , we obtain,

$$
\sigma=\sigma_{0}+\sigma_{01} / X
$$

with,

$$
\begin{aligned}
\sigma_{0}=\left\{\ln \frac{\left(1-v_{\mathrm{p}(1)}\right)}{\left(1-v_{\mathrm{p}(2)}\right)}+\left(v_{\mathrm{p}(2)}-v_{\mathrm{p}(1)}\right)+\left(\frac{v_{\mathrm{p}(2)}}{X_{n(2)}}-\frac{v_{\mathrm{p}(1)}}{X_{n(1)}}\right)\right\} \\
{\left[2\left(v_{\mathrm{p}(2)}-v_{\mathrm{p}(1)}\right)+1.5 p\left(v_{\mathrm{p}(2)}^{2}-v_{\mathrm{p}(1)}^{2}\right)+k\left\{\left(\frac{v_{\mathrm{p}(2)}}{X_{n(2)}}-\frac{v_{\mathrm{p}(1)}}{X_{n(1)}}\right)+p\left(\frac{v_{\mathrm{p}(2)}^{2}}{X_{n(2)}}-\frac{v_{\mathrm{p}(1)}^{2}}{X_{n(1)}}\right)\right\}\right] / } \\
{\left[\left(v_{\mathrm{p}(2)}^{2}-v_{\mathrm{p}(1)}^{2}\right)+p\left(v_{\mathrm{p}(2)}^{3}-v_{\mathrm{p}(1)}^{3}\right)+k\left\{\left(\frac{v_{\mathrm{p}(2)}^{2}}{X_{n(2)}}-\frac{v_{\mathrm{p}(1)}^{2}}{X_{n(1)}}\right)+p\left(\frac{v_{\mathrm{p}(2)}^{3}}{X_{n(2)}}-\frac{v_{\mathrm{p}(1)}^{3}}{X_{n(1)}}\right)\right\}\right]-\ln \frac{\left(1-v_{\mathrm{p}(1)}\right)}{\left(1-v_{\mathrm{p}(2)}\right)} }
\end{aligned}
$$

and

$$
\sigma_{01}=\frac{k\left\{\ln \frac{\left(1-v_{\mathbf{p}(1)}\right)}{\left(1-v_{\mathbf{p}(2)}\right)}+\left(v_{\mathbf{p}(2)}-v_{\mathbf{p}(1)}\right)+\left(\frac{v_{\mathbf{p}(2)}}{X_{n(2)}}-\frac{v_{\mathrm{p}(1)}}{X_{n(1)}}\right)\right\}\left\{\left(v_{\mathrm{p}(2)}-v_{\mathrm{p}(1)}\right)+0.5 p\left(v_{\mathrm{p}(2)}^{2}-v_{\mathrm{p}(1)}^{2}\right)\right\}}{\left(v_{\mathrm{p}(2)}^{2}-v_{\mathrm{p}(1)}^{2}\right)+p\left(v_{\mathbf{p}(2)}^{3}-v_{\mathrm{p}(1)}^{3}\right)+k\left\{\left(\frac{v_{\mathrm{p}(2)}^{2}}{X_{n(2)}}-\frac{v_{\mathbf{p}(1)}^{2}}{X_{n(1)}}\right)+p\left(\frac{v_{\mathbf{p}(2)}^{3}}{X_{n(2)}}-\frac{v_{\mathbf{p}(1)}^{3}}{X_{n(1)}}\right)\right\}}
$$

Putting $k=0$ in eq $12-14$, we obtain the equations derived previously by Kamide and Sugamiya ${ }^{8}$ (eq 15 of ref 8). Further, eq $12-14$ with $k=0$ and $p=0$ reduces the corresponding equations (eq 8 of ref 9 ) in the Flory-Huggins theory.

In summary, eq 4, 5, and 10 furnish us with the theoretical basis for calculating the phaseseparation phenomena, when the $\chi$-parameter is a

$$
\text { function of } X \text { and } v_{\mathbf{p}} \text {. }
$$

\section{COMPUTER SIMULATION}

The mathematical phase separation and successive precipitational and successive solutional fractionations (SPF and SSF) were undertaken according to a simulative procedure established by 
Table I. Molecular characteristics of the original polymer and operating conditions of phase-separation employed in computer simulation

1 Molecular weight distribution

2 Ratio of weight- to number-average molecular weight $\left(X_{w}{ }^{0} / X_{n}{ }^{0}\right)$

3 Weight average of $X\left(X_{w}{ }^{0}\right)$

4 Initial polymer volume fraction

5 Fraction size $\left(\rho_{\mathrm{p}}\right)$

6 Total number of fractions $\left(n_{\mathbf{t}}\right)$

7 Parameter $k$

8 Parameter $p$
Schulz-Zimm distribution

2.8 (for Styron 666)

1810 (in methylcyclohexane)

2120 (in cyclohexane)

$0.47,0.94$, and $1.89 \%$

$0.04-0.92$

2, 18 (SPF), and 20 (SSF)

$0-10$

$0-1.0$ modifying the Kamide and Sugamiya method. ${ }^{8}$ The molecular characreristics of the original polymer and the operating conditions employed are summerized in Table I. The majority of these are just the same as those found in actual experiments on polystyrene in cyclohexane and in methylcyclohexane. $^{2}$

The computer simulation was performed by an electronic computer IBM system 370-158 over the range of $X$ from 15 to 22500 . The original polymer was approximated by 1500 components all differing in $X$. Note that the fraction size is equivalent to the amount of polymer in a phase relative to the original polymer and that, for the polymer remaining in the polymer-rich phase, the fraction size is expressed by $\rho_{\mathrm{p}}$ and for the polymer in the polymer-lean phase, fraction size is expressed by $\rho_{\mathrm{s}}$. The flow sheet of the simulation is illustrated in Figure 1. The symbols in Figure 1 have the same meaning as those in ref 8 .

\section{RESULTS AND DISCUSSION}

The partition coefficient $\sigma$ was calculated by assuming $k=1 \sim 10$ under operating conditions identical to those employed in the previous paper ${ }^{2}$ for PS-CH and PS-MCH systems: The original polymer, Schulz-Zimm distribution with $X_{w}^{0} / X_{n}^{0}=2.8 \quad\left(X_{w}^{0}=2120\right.$ for PS-CH and $X_{w}{ }^{0}=1810$ for PS-MCH); $p=0.6$ (for PS-CH) and 0.7 (for PS-MCH); initial polymer volume fraction, $v_{\mathrm{p}}{ }^{0}=0.47-1.89 \mathrm{vol} \%$; the fraction size $\rho_{\mathrm{p}}=0.04$ 0.92 , where $X_{w}{ }^{0}$ and $X_{n}{ }^{0}$ are the weight- and number-average of $X$ of the original polymer. In Figure 2a)-2d), the plot of $\sigma$ as a function of $1 / X$ is illustrated. The open circles are experimental points for the PS-CH (2a) and PS-MCH (2b-2d) systems, and the number attached to each line indicates the $k$ value. As $k$ gets larger, the slope of the plot, i.e., $\sigma_{01}$ (eq 12), increases. For relatively small $\rho_{\mathrm{p}}(0.04$ $0.48)$, the experimental points are not far from the theoretical lines and there exists a one-to-one correspondence between $k$ and $\sigma_{01}$ at constant $\rho_{\mathrm{p}}$, but for $\rho_{\mathrm{p}},(0.52-0.92)$ the values of theoretical $\sigma$ differ significantly from the measured values. The slope of the experimental plot enables us to estimate $\sigma_{01}$. These results are summerized in Table II, where it is shown that $\sigma_{01}$ lies between 0.12 and 1.06 for the PS-CH system and between -0.67 and 1.28 for the PS-MCH system. The theoretical relationships between $\sigma_{01}$ and the $k$-parameter at a given $\rho_{\mathrm{p}}$, estimated from the theoretical $\sigma v s .1 / X$ plots, are shown in Figure 3a) for PS-CH and $3 b-d$ ) for PS-MCH, where the number on each curve denotes the value of $\rho_{\mathrm{p}}$. Log-Log plots of $\sigma_{01} v s . k$ can be represented by a straight line throughtout the entire range of $k$ investigated. $\sigma_{01}$ increases noticeablly with increasing $k$ and $\rho_{\mathrm{p}}$. The $k$ values evaluated from $\sigma_{01}$ in Table II, by using the relations in Figure 3, are collected in the eight column of the table. The $k$ parameter was found to be $1.8-7.9$ for PS- $\mathrm{CH}$ and 3.7-8.7 for PS-MCH. In this manner, we determined $k$ from the experimentally obtained molecular weight dependence of the partition coefficient $\sigma$. Conversely, the molecular weight dependence of $\sigma$ can, at least partially, be interpreted by the molecular weight dependence of the $\chi$-parameter. Obviously, all the $k$ values, except two, estimated by this method are positive.

If the $\chi$-parameter is experimentally determined as a function of molecular weight and concentration for a given polymer-solvent system at a given temperature, the data afford us with another route for evaluating $k$. Scholte $\mathrm{e}^{10,11}$ used the light- 


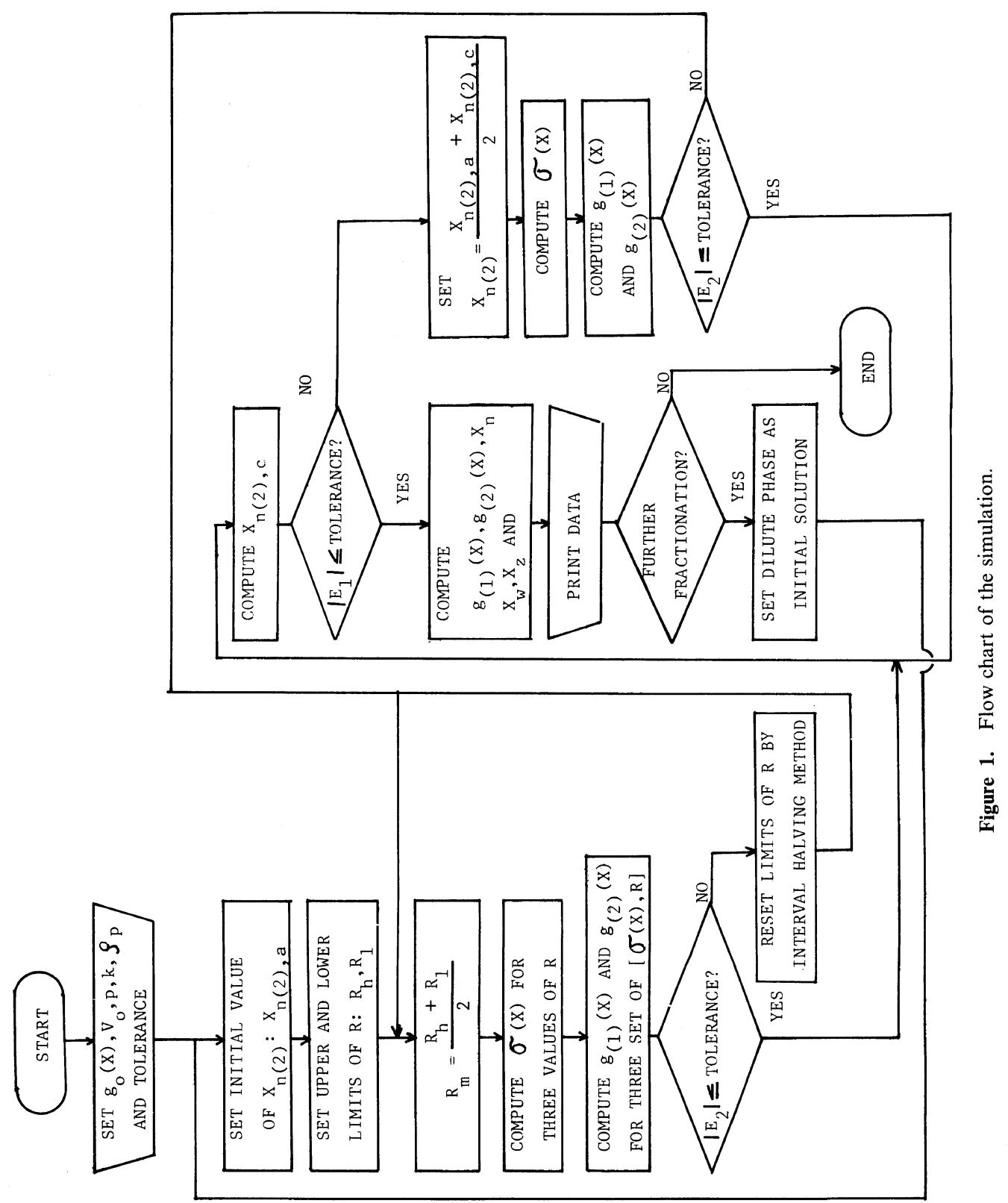




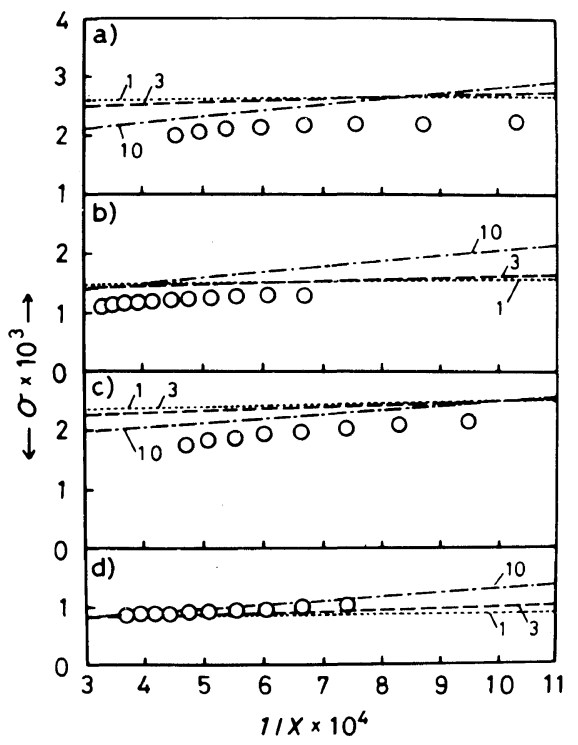

Figure 2. Plot of the partition coefficient, $\sigma$, versus the reciprocal of the molar volume ratio of polymer to solvent, $1 / X$ : open circle, experimental data for polystyrene (the Schulz-Zimm (SZ) distribution, $M_{w}=23.2 \times$ $\left.10^{4}, M_{n}=8.9 \times 10^{4}\right)$ in cyclohexane $(\mathrm{CH})(\mathrm{a})$ and methylcyclohexane $(\mathrm{MCH})(\mathrm{b})-(\mathrm{d})$; broken and chain lines, theoretical curves calculated assuming $k=1,3$, and 10 under the conditions identical with those in actual experiments ( $\mathrm{SZ}$ distribution, $X_{w}{ }^{0} / X_{n}{ }^{0}=2.8, X_{w}{ }^{0}=2120$ in $\mathrm{CH}$ and 1810 in $\mathrm{MCH}, p=0.6$ in $\mathrm{CH}$ and 0.7 in $\mathrm{MCH})$; a) PS-CH, $v_{\mathrm{p}}{ }^{0}=0.97 \mathrm{vol}_{\mathrm{o}} \%, \rho_{\mathrm{p}}=0.25$; b) PS$\left.\mathrm{MCH}, v_{\mathrm{p}}{ }^{0}=0.47 \mathrm{vol} \%, \rho_{\mathrm{p}}=0.10 ; \mathrm{c}\right)$ PS-MCH, $v_{\mathrm{p}}{ }^{0}=0.94$ $\mathrm{vol}^{\circ} \%, \rho_{\mathrm{p}}=0.48$; d) PS-MCH, $v_{\mathrm{p}}{ }^{0}=1.89 \mathrm{vol} \%, \rho_{\mathrm{p}}=0.07$.

Table II. Parameter $\chi_{01}, \sigma_{01}, p$, and $k$

\begin{tabular}{|c|c|c|c|c|c|c|c|}
\hline \multirow{2}{*}{$\begin{array}{c}\text { Polymer- } \\
\text { solvent }\end{array}$} & \multirow{2}{*}{$\frac{v_{\mathrm{p}}^{0}}{\%}$} & \multirow{2}{*}{$\rho_{\mathrm{p}}$} & \multirow{2}{*}{$\frac{\text { Temp }}{{ }^{\circ} \mathrm{C}}$} & \multirow{2}{*}{$\chi_{01}$} & \multirow{2}{*}{$\sigma_{01}$} & \multirow{2}{*}{$p$} & \multirow{2}{*}{$k$} \\
\hline & & & & & & & \\
\hline \multirow[t]{8}{*}{ PS-CH } & 0.94 & 0.88 & 10.7 & - & 1.06 & $(0.6)$ & 6.7 \\
\hline & & 0.84 & 15.0 & - & 1.05 & (0.6) & 7.9 \\
\hline & & 0.62 & 20.0 & - & 0.61 . & $(0.6)$ & 6.3 \\
\hline & & 0.25 & 24.0 & - & 0.12 & $(0.6)$ & 1.8 \\
\hline & - & - & 30 & 0.5009 & - & 0.62 & -0.55 \\
\hline & - & - & 35 & 0.4993 & - & 0.64 & -0.87 \\
\hline & - & - & 45 & 0.4899 & - & 0.72 & -0.67 \\
\hline & - & - & 65 & 0.4840 & - & 0.50 & -12.3 \\
\hline \multirow[t]{12}{*}{ PS-MCH } & 0.47 & 0.88 & 20.0 & - & 0.79 & $(0.7)$ & 3.7 \\
\hline & & 0.78 & 38.0 & - & 1.13 & $(0.7)$ & 8.7 \\
\hline & & 0.59 & 45.7 & - & 0.96 & $(0.7)$ & 8.6 \\
\hline & & 0.10 & 54.9 & - & 0.63 & $(0.7)$ & 7.6 \\
\hline & 0.94 & 0.92 & 20.0 & - & 1.00 & $(0.7)$ & 5.0 \\
\hline & & 0.59 & 47.9 & - & 0.93 & $(0.7)$ & 4.5 \\
\hline & & 0.48 & 50.0 & - & 0.65 & $(0.7)$ & 4.7 \\
\hline & & 0.04 & 57.5 & - & -0.67 & $(0.7)$ & - \\
\hline & 1.89 & 0.92 & 24.0 & - & -0.37 & $(0.7)$ & - \\
\hline & & 0.35 & 54.6 & - & 1.28 & $(0.7)$ & 6.8 \\
\hline & & 0.31 & 57.9 & - & 0.54 & $(0.7)$ & 6.4 \\
\hline & & 0.07 & 56.1 & - & 0.43 & $(0.7)$ & 6.4 \\
\hline
\end{tabular}

scattering method to determine the $\chi$-parameter for PS samples having different molecular weights in CH. Using his experimental data, (Table I of ref 10 and Tables I, II, and III of ref 11), we determined $p$ and $k$ for the PS-CH system by the regression method. The results are shown in Table II. It should be noted that these $k$ values above Flory's $\theta$-temperature are negative.

The temperature dependence of $k$ for the PS-CH and PS-MCH systems is shown in Figure 4. The data points evaluated by analyzing two different experiments by two different methods fall approx- 
imately on a single curve. This implies that the values of the $k$-parameter, estimated from the partition coefficient, are acceptable as a first

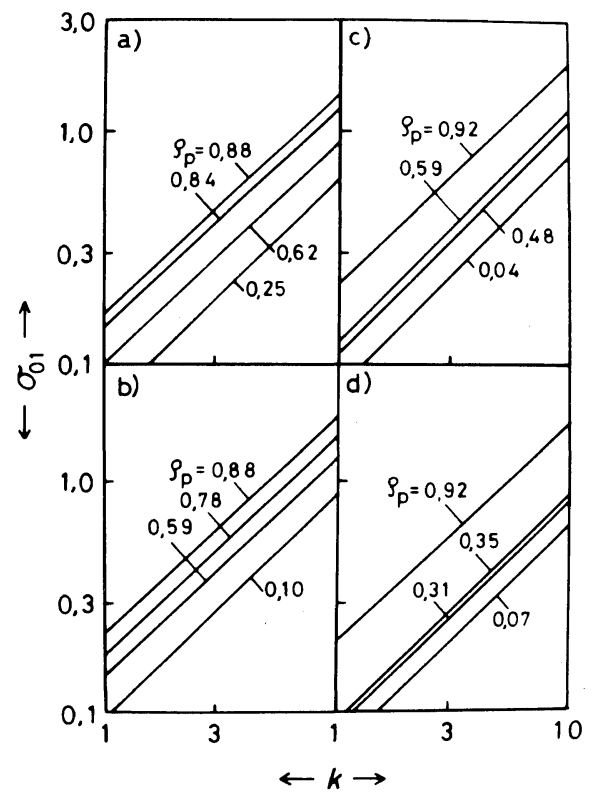

Figure 3. Relationships between $\sigma_{01}$ and the $k$ parameter: a) the original polymer (Schulz-Zimm (SZ) distribution, $\left.X_{w}{ }^{0} / X_{n}{ }^{0}=2.8, X_{w}{ }^{0}=2120\right), v_{\mathrm{p}}{ }^{0}=0.94 \mathrm{vol} \%$, $p=0.6$; b) the original polymer (SZ distribution $\left.X_{w}{ }^{0} / X_{n}{ }^{0}=2.8, X_{w}{ }^{0}=1810\right), v_{\mathrm{p}}{ }^{0}=0.47 \mathrm{vol} \%, p=0.7$; $)$ the original polymer (SZ distribution, $X_{w}{ }^{0} / X_{n}^{0}=2.8$, $\left.\left.X_{w}{ }^{0}=1810\right), v_{\mathrm{p}}{ }^{0}=0.94 \mathrm{vol} \%, p=0.7 ; \mathrm{d}\right)$ the original polymer (SZ distribution, $X_{w}{ }^{0} / X_{n}{ }^{0}=2.8, X_{w}{ }^{0}=1810$ ), $v_{\mathrm{p}}{ }^{0}=1.89 \mathrm{vol}_{\mathrm{o}}^{\mathrm{o}}, p=0.7$. The value of $\rho_{\mathrm{p}}$ is indicated on curve: a) corresponds to PS-CH and b) $-\mathrm{d}$ ) to PS$\mathrm{MCH}$. approximation. Evidently, $k$ decreases with an increase in temperature. In order to evaluate $k$ with two significant figures, we need $\chi$ values accurate to four significant digits when the polymer samples have comparatively large $M_{n}$ as in the case of Scholte's study $\left[M_{n}=49,000\left(X_{n}=433\right), 154,000\right.$ $\left(X_{n}=1363\right)$ and $\left.435,000\left(X_{n}=3550\right)\right]$. Such precision seems undoubtedly beyond the accuracy of actual experiments at present. On the basis of Krigbaum's experiments ${ }^{12}$ on the second virial coefficient $A_{2}$ for five polystyrene samples in cyclohexane, Koningsveld and Kleintjens ${ }^{6}$ speculated a change in the sign of molecular weight dependence of the $\chi$ parameter in the vicinity of $\theta$-temperature and predicted a positive value of $k$ from a comparison of the data of spinodal with the theory for the PS-CH system. Figure 4 confirms the validity of their prediction. Thus in order to discuss the effect of the molecular weight dependence of the $\chi$-parameter on fractionation by solubility, the $k$ value in the temperature range, wherein the phase-separation experiment is carried out, should be utilized in place of that obtained from the $\chi$-parameter above the $\theta$ temperature.

Figures 5-7 illustrate the effect of $k$ on the molecular weight distributions (MWD) of polymer in the polymer-rich phase [a), b) and c)] and the polymer-lean phase [d), e), and f)] for the PS- $\mathrm{MCH}$ system. The full line is the experimental MWD curve evaluated by gel-permeation chromatography in the previous paper ${ }^{2}$ and the dotted, broken and chain lines are the theoretical curves calculated assuming $k=0,3$, and 10 , respectively, under the same conditions as in the experiments.

Table III. The $k$-parameter in eq 4 evaluated for polystyrene-cyclohexane and polystyrene-methylcyclohexane system

\begin{tabular}{|c|c|c|c|c|}
\hline \multirow{2}{*}{ No. } & \multirow{2}{*}{ Method } & \multicolumn{2}{|c|}{$k$-parameter } & \multirow{2}{*}{ Remarks } \\
\hline & & PS-CH & PS-MCH & \\
\hline 1 & $\begin{array}{l}\text { Molecular weight dependence of the partition } \\
\text { coefficient }\end{array}$ & $1.8-7.9$ & $3.7-8.7$ & Table II \\
\hline 2 & Molecular weight dependence of the $\chi$-parameter & $-0.55-12.3$ & - & Table II \\
\hline 3 & $\begin{array}{l}\text { Molecular weight distribution of polymer } \\
\text { in a polymer-rich phase or a polymer-lean phase }\end{array}$ & $3-10$ & $3-10$ & $\begin{array}{l}\text { Figures } \\
\qquad 4-6\end{array}$ \\
\hline 4 & $\begin{array}{l}\text { The ratio } M_{w} / M_{n} \text { of the fraction } \\
\text { (in this case, SPF) }\end{array}$ & - & $0-10$ & Figure 17 \\
\hline 5 & $\begin{array}{l}\text { Plot of the ratio } M_{w} / M_{n} \text { against } M_{w} \text { of the } \\
\text { fractions obtained by a SPF run }\end{array}$ & - & $1-3$ & Figure 19 \\
\hline
\end{tabular}




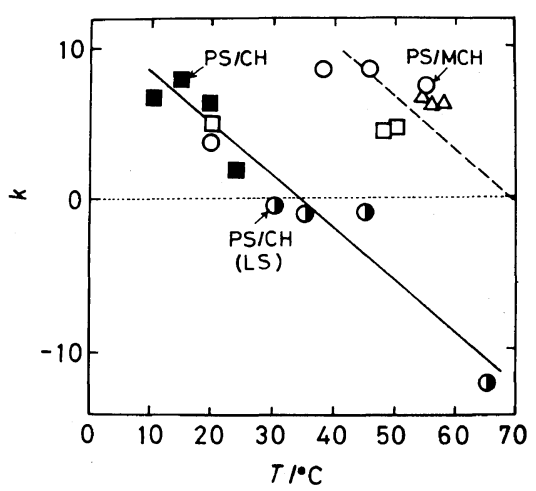

Figure 4. Change in the $k$-parameter with temperature: closed mark, PS-CH system; open mark, PS-MCH system; $\bigcirc, v_{\mathrm{p}}{ }^{0}=0.47 \mathrm{vol} \% ; \square, v_{\mathrm{p}}{ }^{0}=0.94 \mathrm{vol} \% ; \triangle$, $v_{\mathrm{p}}{ }^{0}=1.89 \mathrm{vol} \% ; \boldsymbol{O}$, data of Scholte by light-scattering method. ${ }^{9,10}$ Full line was drown by fitting the experimental data with use of the least square method for PS- $\mathrm{CH}$ system and broken line was drown intuitively in parallel with the full line for PS-MCH system.

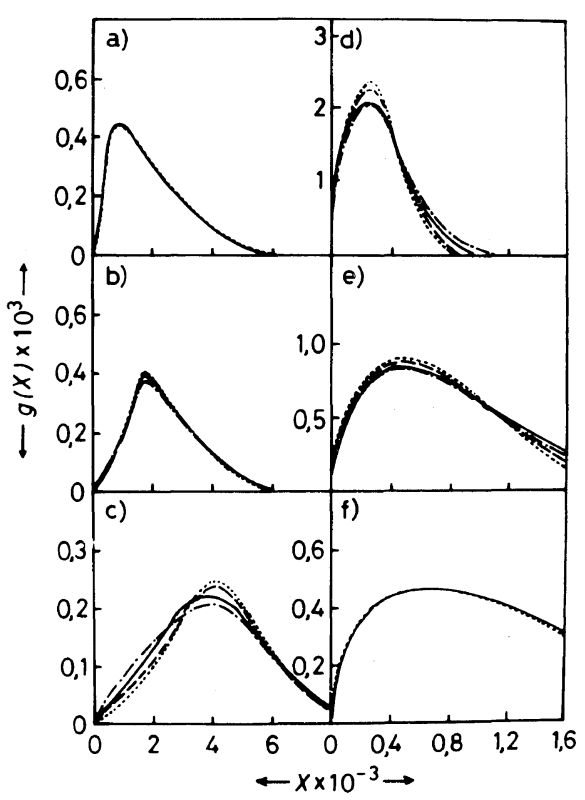

Figure 5. Molecular weight distribution curve, $g(X)$, of the polymers in a polymer-rich phase [a)-c)] and a polymer-lean phase [d)—f)], separated from a $0.47 \mathrm{vol} \%$ of PS solution in $\mathrm{MCH}$ : a) $\rho_{\mathrm{p}}=0.88$; b) $\rho_{\mathrm{p}}=0.59$; c) $\rho_{\mathrm{p}}=0.10$; d) $\rho_{\mathrm{s}}=0.12$; e) $\rho_{\mathrm{s}}=0.41$; f) $\rho_{\mathrm{s}}=0.90$; full line, experimental curve ${ }^{2}$; dotted, broken, and chain lines, theoretical curves; $k=0,3$, and 10 .

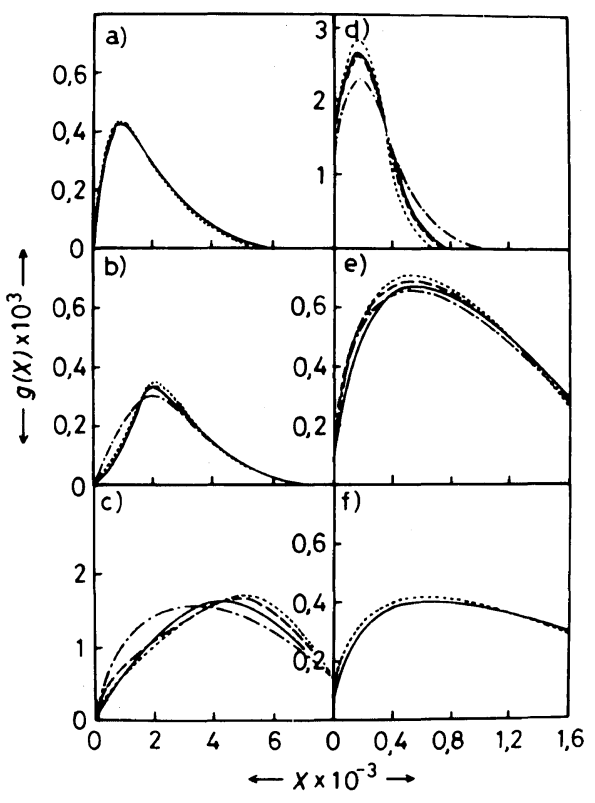

Figure 6. Molecular weight distribution curve, $g(X)$, of the polymer-rich phase [a) - c)] and a polymer-lean phase [d) - f )], both separated from a $0.94 \mathrm{vol} \%$ of PS solution in MCH: a) $\rho_{\mathrm{p}}=0.92$; b) $\rho_{\mathrm{p}}=0.48$; c) $\rho_{\mathrm{p}}=0.04$; d) $\rho_{\mathrm{s}}=0.08$; e) $\rho_{\mathrm{s}}=0.52$; f) $\rho_{\mathrm{s}}=0.96$; full line, experimental curve ${ }^{2}$; dotted, broken, and chain lines, theoretical curves with $k=0,3$, and $10, \mathrm{a}), \mathrm{b})$, and c) are counterparts of $d, e)$, and $f$ ).

The MWD curves in the polymer-rich phase at small $\rho_{\mathrm{p}}$ and that in the polymer-lean phase at small $\rho_{\mathrm{s}}$ undergo rapid change, becoming markedly sharp as the $k$-parameter decreases. In other words, if the fraction size is small as in the case of practical fractionation, the effect of the $k$-parameter on the MWD of the fractions isolated by SPF and SSF is considerably large, over a wide range of the initial polymer volume fraction of the original solution. The MWD in the polymer-rich phase at large $\rho_{\mathrm{p}}$ and that in the polymer-lean phase at large $\rho_{\mathrm{s}}$ are not very sensitive to the $k$-parameter. Thus, there is a possibility of estimating the magnitude of the $k$ parameter by a comparison of the MWD curves of the polymer with very small $\rho_{\mathrm{s}}$ or $\rho_{\mathrm{p}}$ between theory and experiment. For the former (small $\rho_{\mathrm{s}}$ ), the $p$ parameter is not prerequisite, but for the latter (small $\rho_{\mathrm{p}}$ ), the $p$-parameter is needed in advance, as will be demonstrated later. In this case, particular difficulties are involved in determining the molecular weight distribution for a lower molecular weight 


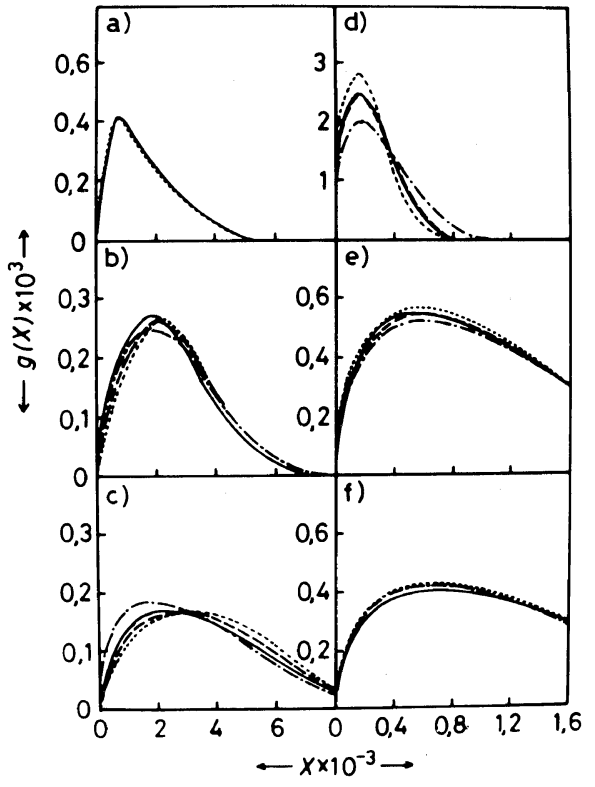

Figure 7. Molecular weight distribution curve, $g(X)$, of the polymers in a polymer-rich phase [a) - c)] and a polymer-lean phase [d) - f)], both separated from a 1.89 $\mathrm{vol}^{\circ} \%$ of PS solution in MCH: a) $\rho_{\mathrm{p}}=0.92$; b) $\rho_{\mathrm{p}}=0.35$; c) $\rho_{\mathrm{p}}=0.07$; d) $\rho_{\mathrm{s}}=0.08$; e) $\rho_{\mathrm{s}}=0.65$; f) $\rho_{\mathrm{s}}=0.93$; full line, experimental curve ${ }^{2}$; dotted, broken, and chain. lines, theoretical curves with $k=0,3$, and 10 .

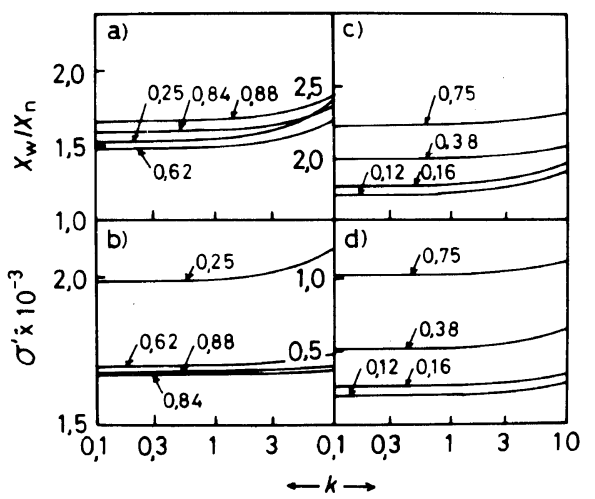

Figure 8. Theoretical dependence of the ratio $X_{w} / X_{n}$ and the standard deviation $\sigma^{\prime}$ of the polymers in a polymer-rich phase $[a), b)]$ or in a polymer-lean phase $[\mathrm{c}), \mathrm{d})]$ on the $k$-parameter; the original polymer (Schulz-Zimm (SZ) distribution, $X_{w}{ }^{0} / X_{n}{ }^{0}=2.8, X_{w}{ }^{0}=$ 2120); $v_{\mathrm{p}}{ }^{0}=0.94 \mathrm{vol} \% ; p=0.6$. $\rho_{\mathrm{p}}$ or $\rho_{\mathrm{s}}$ is shown on curve; a) -d) correspond to actual experiments on PS-MCH system.

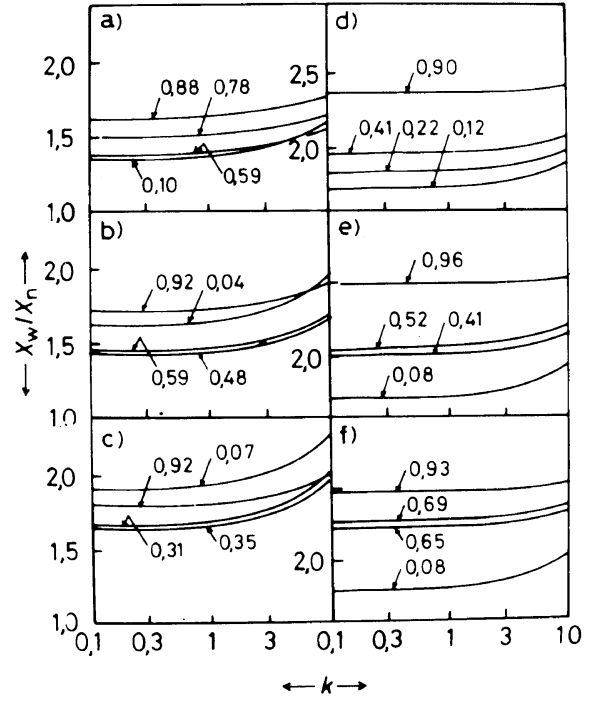

Figure 9. Theoretical dependence of the ratio $X_{w} / X_{n}$ of the polymers in a polymer-rich phase [a) - c)] or in a polymer-lean phase [d)-f)] on the $k$-parameter; the original polymer (Schulz-Zimm (SZ) distribution, $\left.X_{w}{ }^{0} / X_{n}{ }^{0}=2.8, X_{w}{ }^{0}=1810\right) ; v_{\mathrm{p}}{ }^{0}=0.47 \mathrm{vol} \%$ (a) and d)); $0.94 \mathrm{vol} \%$ (b) and e)), and $1.89 \mathrm{vol} \%$ (c) and f)); $p=0.7$. $\rho_{\mathrm{p}}$ or $\rho_{\mathrm{s}}$ is indicated on curve; a) -f) correspond to actual experiments on the PS-MCH system.

and (if necessary) to express this by an adequate analytical function. These difficulties are probably enhanced when determining the partition coefficient from the ratio of the weight fractions of a given component in the two phases. Figures 6 and 7 reveal that the experimental MWD curves at small $\rho_{\mathrm{p}}$ or $\rho_{\mathrm{s}}$ are in good agreement with the theoretical curves with $k=3-10$ and the experimental MWD curves at large $\rho_{\mathrm{p}}$ or $\rho_{\mathrm{s}}$ fit, as the theory predicts, exactly the theoretical curves, irrespective of $k$, at least ranging 0 to 10 . The latter verifies the high reliability of the actual experiments in the previous paper, indicating that the difference between the experimental and theoretical MWD curves for $k=0$ cannot be attributed to experimental error.

In Figures $8-10$ are shown the theoretical relations between the ratio $X_{w} / X_{n}$ or the standard deviation $\sigma^{\prime}$ of the polymer in either the polymerrich or the polymer-lean phase and the $k$-parameter for the PS-CH and PS-MCH systems. Here, calculations were performed for polymers having the same molecular characteristics under the same operating condition as those employed in the actual 


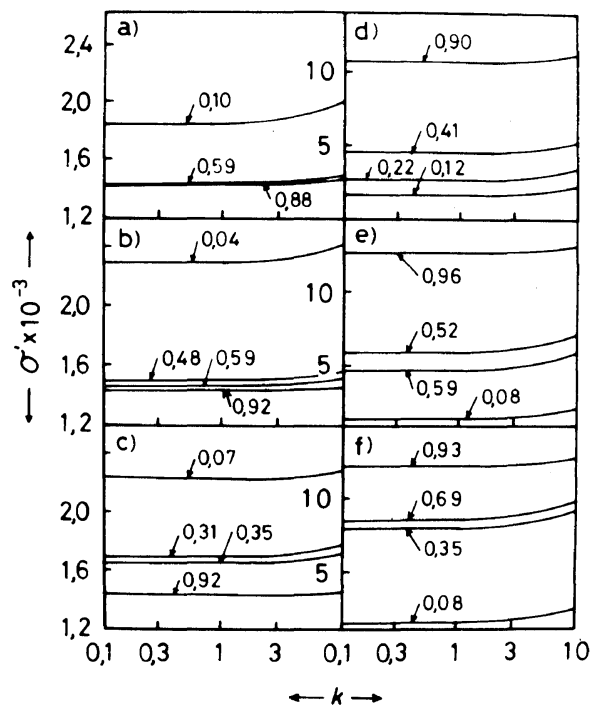

Figure 10. Theoretical dependence of the standard deviation $\sigma^{\prime}$ of the molecular weight distribution of the polymers in a polymer-rich phase [a)-c)] or in a polymer-lean phase [d)- $\mathrm{f}$ )] on the $k$-parameter; the original polymer (Schulz-Zimm (SZ) distribution, $X_{w}{ }^{0} / X_{n}{ }^{0}=1810 ; v_{\mathrm{p}}{ }^{0}=0.47 \mathrm{vol} \%$ [a) and d)], $0.94 \mathrm{vol}^{\circ} \%$ [b) and e)], and $1.89 \mathrm{vol} \%$ [c) and f)]; $p=0.7, \rho_{\mathrm{p}}$ or $\rho_{\mathrm{s}}$ is indicated on curve; a)-f) correspond to actual experiments on the PS-MCH system.

phase-separation experiments: The original polymer, the Schulz-Zimm distribution $\left(X_{w}{ }^{0}=1810\right.$, $\left.X_{w}{ }^{0} / X_{n}{ }^{0}=2.8\right)$; initial polymer volume fraction, $v_{\mathrm{p}}{ }^{0}=0.47,0.94$, and $1.89 \mathrm{vol}^{\%} \%$; the fraction size, $\rho_{\mathrm{p}}=0.04-0.92$. The positive molecular weight dependence of the $\chi$-parameter (i.e., $k>0$ ) makes the polymolecularity of the polymers in the two phases unquestionably larger, particularly in the range of $k>3$. Therefore, from a experimental point of view, $M_{w} / M_{n}$ of the polymers in the two phases can be regarded as independent of the $k$-parameter if the $k$ value for polymer-solvent combination does not exceed 3. A comparision of the theoretical curves on $M_{w} / M_{n}-k-\rho_{\mathrm{p}}$ with the experiments will be given later (see, Figure 18).

In previous papers ${ }^{8,13-16}$ it was confirmed that a large positive concentration dependence parameter $p$ in eq 1 makes polydispersity of the fractions small. Accordingly, small a positive or negative $p$ as well as a large $k$ may give rise to a broad-distribution fraction, provided that the other parameters are the same. In order to clarify this expectation, similar

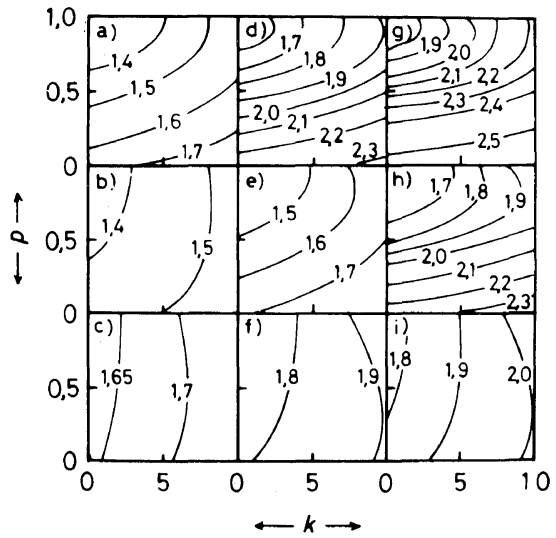

Figure 11. Theoretical correlations between the $p$ parameter, the $k$-parameter and the ratio $X_{w} / X_{n}$ of the polymer partitioned in a polymer-rich phase: the original polymer (Schulz-Zimm distribution, $X_{w}{ }^{0} / X_{n}{ }^{0}=2.8$, $\left.X_{w^{\prime}}{ }^{0}=1810\right)$;

a) $v_{\mathrm{p}}{ }^{0}=0.47 \mathrm{vol} \%, \rho_{\mathrm{p}}=0.10$;

b) $v_{\mathrm{p}}{ }^{0}=0.47 \mathrm{vol} \%, \rho_{\mathrm{p}}=0.59$;

c) $v_{\mathrm{p}}{ }^{0}=0.47 \mathrm{vol} \%, \rho_{\mathrm{p}}=0.88$

d) $v_{\mathrm{p}}{ }^{0}=0.94 \mathrm{vol}^{\circ} / \rho_{\mathrm{p}}=0.04$;

e) $v_{\mathrm{p}}{ }^{0}=0.94 \mathrm{vol} \%, \rho_{\mathrm{p}}=0.48$;

f) $v_{\mathrm{p}}{ }^{0}=0.94 \mathrm{vol} \%, \rho_{\mathrm{p}}=0.92$;

g) $v_{\mathrm{p}}{ }^{0}=1.89 \mathrm{vol} \%, \rho_{\mathrm{p}}=0.07$;

h) $v_{\mathrm{p}}^{0}=1.89 \mathrm{vol}^{0} \%, \rho_{\mathrm{p}}=0.35$;

i) $v_{\mathrm{p}}{ }^{0}=1.89 \mathrm{vol} \%, \rho_{\mathrm{p}}=0.92$.

computer simulations were performed under the same operating conditions except for parameters $p$ and $k$, as those employed in Figures $5-8$. In this case, the molecular weight and concentration dependences of the $\chi$-parameter (i.e., $k$ - and $p$ parameters) were varied concurrently in the ranges: $0 \leqq k \leqq 10$ and $0 \leqq p \leqq 1$. The relationships between $p$ and $k$, which yield a given $M_{w} / M_{n}$ value of polymers in either the polymer-rich phase or the polymer-lean phase, was estimated using the fractionation data thus calculated. The results are shown in Figures 11 and 12 . For the polymers with small $\rho_{\mathrm{p}}$ remaining in the polymer-rich phase, the $M_{\mathrm{w}} / M_{n}$ values are remarkably influenced by both $p$ and $k$ [Figure $11 \mathrm{a}$ ), d) and g)], especially when the phase separation occures from a relatively concentrated solution [Figure 11]. In general, a large positive molecular weight dependence and a small positive (or negative) concentration dependence of the $\chi$ parameter (large $k$ and small $p$ ) afford us with fractions having large polymolecularity, as expected. In contrast to this, in a large $\rho_{\mathrm{p}}$ region, the 


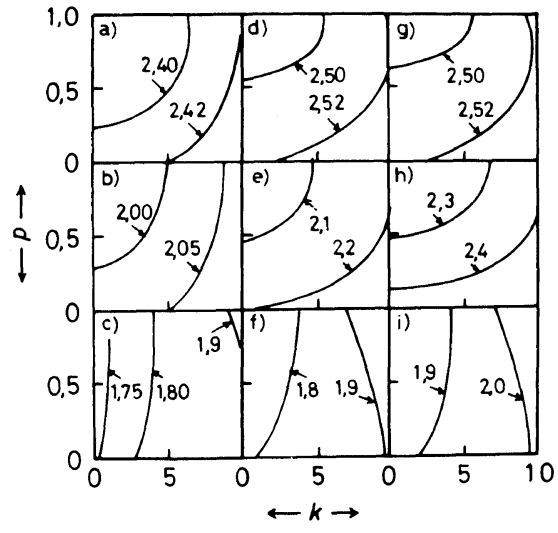

Figure 12. Theoretical correlations between the $p$ parameter, the $k$-parameter, and the ratio $X_{w} / X_{n}$ of the polymer remained in a polymer-lean phase: the original polymer (Schulz-Zimm distribution, $X_{w}{ }^{0} / X_{n}{ }^{0}=2.8$, $X_{w}{ }^{0}=1810$ );
a) $v_{\mathrm{p}}^{0}=0.47 \mathrm{vol}^{\mathrm{o}} \%, \rho_{\mathrm{s}}=0.90$
b) $v_{\mathrm{p}}{ }^{0}=0.47 \mathrm{vol} \%, \rho_{\mathrm{s}}=0.53$;
c) $v_{\mathrm{p}}^{0}=0.47 \mathrm{vol} \%, \rho_{\mathrm{s}}=0.12$
d) $v_{\mathrm{p}}{ }^{0}=0.94 \mathrm{vol}^{\circ} \%, \rho_{\mathrm{s}}=0.96$;
e) $v_{\mathrm{p}}{ }^{0}=0.94 \mathrm{vol} \%, \rho_{\mathrm{s}}=0.52$
f) $v_{\mathrm{p}}{ }^{0}=0.94 \mathrm{vol}^{\circ} \%, \rho_{\mathrm{s}}=0.08$;
g) $v_{\mathrm{p}}{ }^{0}=1.89 \mathrm{vol} \%, \rho_{\mathrm{s}}=0.93$;
h) $v_{\mathrm{p}}{ }^{0}=1.89 \mathrm{vol} \%, \rho_{\mathrm{s}}=0.65$;
i) $v_{\mathrm{p}}{ }^{0}=1.89 \mathrm{vol} \%, \rho_{\mathrm{s}}=0.08$.

$M_{w} / M_{n}$ of the precipitate depends markedly on the $k$ value rather than on the $p$ value. Note that the $k$ parameter dependence of the polydispersity of the polymer partitioning in both phases, as shown in Figures $8-10$, are calculated assuming $p=0.6$ for PS-CH system and $p=0.7$ for PS-MCH system, respectively. The $M_{w} / M_{n}$ of the polymers in the two phases are closely connected in a much more complex manner with both $p$ - and $k$-parameters.

In order to clarify the effect of $k$ and $p$-parameters on the polydispersity of the polymers partitioning in the two phases, a $1 \mathrm{vol} \%$ of the original polymer, having the Schulz-Zimm molecular weight distribution $\left(X_{w}{ }^{0} / X_{n}^{0}=2\right.$ and $\left.X_{w}{ }^{0}=300\right)$, dissolved in a single solvent, was hypothetically phase separated to give various $\rho_{\mathrm{p}}\left(\equiv 1-\rho_{\mathrm{s}}\right)$. The results are summerized in Figure 13, where a) and b) denote the polymer-rich phase, and c) and d), the polymer-lean phase. Figures 13 a) and c) show the effect of the $k$ parameter at constant $p(=0)$, and Figures $13 \mathrm{~b}$ ) and d) illustrate the effect of the $p$-parameter at constant $k(=0)$. The polydispersity of the polymer in the polymer-rich pahse is influenced approximately

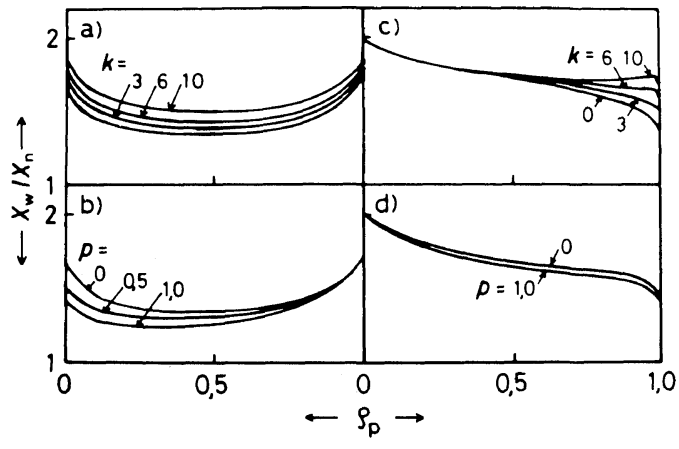

Figure 13. Plot of $X_{w} / X_{n}$ of the polymer in a polymerrich phase [a) and b)] and in a polymer-lean phase [c) and d)]: the original polymer (Schulz-Zimm distribution, $\left.X_{w}{ }^{0} / X_{n}{ }^{0}=2, X_{w}{ }^{0}=300\right)$; a) and c) $p=0, k$-parameter is indicated on the curve; b) and d) $k=0, p$-parameter is indicated on the curve.

equally by the $k$-parameter, and independent of the $\rho_{\mathrm{p}}$ value, but it is markedly effected by the $p$ parameter in the smaller $\rho_{\mathrm{p}}$ region. With an increase in the fraction size $\rho_{\mathrm{p}}$ the $p$-parameter dependence of the polydispersity decreases and approaches zero at the limit of $\rho_{\mathrm{p}}=0.99$ and the $k$-parameter is more predominant than the $p$-parameter in the larger $\rho_{\mathrm{p}}$ $(=0.99)$ region. But its absolute magnitude is too small to be detected experimentally. In other words, the effect of the $p$-parameter on $X_{w} / X_{n}$ of the fraction is larger for smaller $\rho_{\mathrm{p}}$ separated from a more concentrated solution. The effect of the $k$ parameter on the polydispersity of the polymer in the polymer-lean phase becomes significant only in the larger $\rho_{\mathrm{p}}$ (accordingly, smaller $\rho_{\mathrm{s}}$ ) region, and the $p$-parameter lays only a minor role in the polydispersity of the polymer in the polymer-lean phase over an entire $\rho_{\mathrm{s}}$. We have observed a significant effect of the $k$-parameter on the MWD of the polymer in the polymer-rich phase at small $\rho_{\mathrm{p}}$, but it should be noted that this MWD is governed cocurrently by the $p$-parameter.

Previously, Kamide and his coworkers examined systematically the effect of the $p$-parameter on the fractionation characteristics in SPF and SSF when $k=0$ and found that polydispersity of the fractions isolated by SPF and SSF decreases monotonously with an increase in the $p$-parameter. ${ }^{2}$ Figure 14 demonstrates the effect of the $p$-parameter on the $X_{w} / X_{n}$ value of polymers in two phases for PS$\mathrm{MCH}$ system in the small region of $\rho_{\mathrm{p}}$ or $\rho_{\mathrm{s}}$. The curves were calculated by using the same conditions 


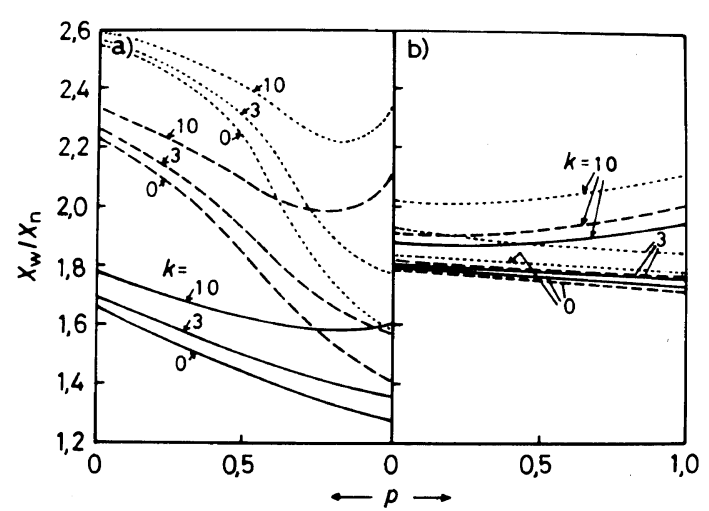

Figure 14. Theoretical dependence of the ratio $X_{w} / X_{n}$ of the polymers in a polymer-rich phase a) and a polymer-lean phase b) on the $p$-parameter for PS-MCH system. The $k$-parameter is indicated on the curve.

a) full line, $v_{\mathrm{p}}{ }^{0}=0.47 \mathrm{vol} \%, \rho_{\mathrm{p}}=0.10$; broken line, $v_{\mathrm{p}}{ }^{0}=0.94 \mathrm{vol} \%, \rho_{\mathrm{p}}=0.04 ;$ dotted line, $v_{\mathrm{p}}{ }^{0}=1.89 \mathrm{vol} \%$, $\rho_{\mathrm{p}}=0.07$.

b) full line, $v_{\mathrm{p}}{ }^{0}=0.47 \mathrm{vol} \%, \rho_{\mathrm{s}}=0.12$; broken line, $v_{\mathrm{p}}{ }^{0}=0.94 \mathrm{vol} \%, \rho_{\mathrm{s}}=0.08 ;$ dotted line, $v_{\mathrm{p}}{ }^{0}=1.89 \mathrm{vol} \%$, $\rho_{\mathrm{s}}=0.08$.

as those in actual experiments, assuming various values for $k$. Obviously, the dependenve of $X_{w} / X_{n}$ on the $p$-parameter is fundamentally altered by the presence of the $k$-parameter, and $X_{w} / X_{n}$ reveals a minimum at a given $p$ value, above which the polydispersity of the fraction increases as the $p$ parameter increases. For a polymer with small $\rho_{\mathrm{s}}$ in the polymer-lean phase, $k$-parameter is more predominate than the $p$-parameter, if the phase separation occurs from a relatively concentrated solution $\left(v_{\mathrm{p}}{ }^{0}>1 \mathrm{vol} \%\right)$. If we adopt the breadth of molecular weight distribution (MWD) in each phase as a measure representing the fractionation efficiency, ${ }^{15}$ the efficiency decreases as $k$ increases.

Equation 1 indicates that the molecular weight dependence of the $\chi$-parameter influences to a lesser extent the fractionation characteristics with an increase in the average molecular weight of the original polymer. In order to confirm this, a series of hypothetical phase-separation calculations were performed under the following conditions, which correspond to actual experiments for PS-MCH system. ${ }^{2}$ Original polymer, the Schulz-Zimm distribution $\left(X_{w}{ }^{0} / X_{n}^{0}=2.8, X_{w}{ }^{0}=150-3000\right) ; v_{\mathrm{p}}{ }^{0}=0.94$ $\mathrm{vol} \%, \rho_{\mathrm{p}}=0.04 \quad\left(\rho_{\mathrm{s}}=0.96\right)$, and $0.92\left(\rho_{\mathrm{s}}=0.08\right)$; $p=0,3$, and 10. Figures 15 a) and $15 \mathrm{~b}$ ) show the theoretical relations between $X_{w}{ }^{0}$ and $X_{w} / X_{n}$ of the

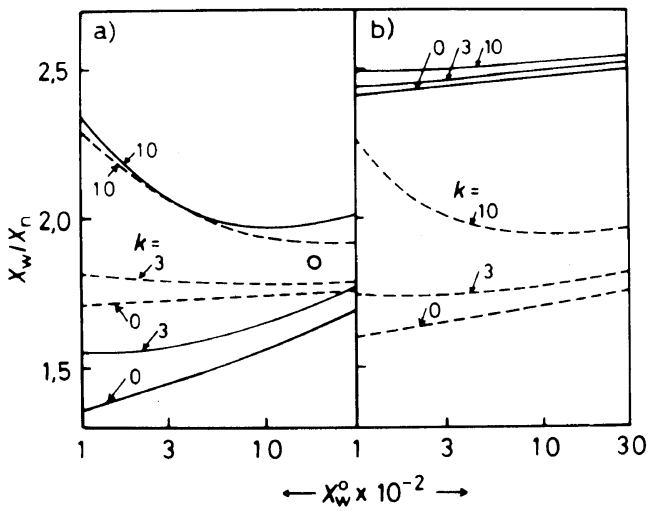

Figure 15. Effect of $X_{w}{ }^{0}$ on the ratio $X_{w} / X_{n}$ of the polymers in a polymer-rich phase (a) and in a polymerlean phase (b): full line, $\rho_{\mathrm{p}}=0.04\left(\rho_{\mathrm{s}}=0.96\right)$; broken line $\rho_{\mathrm{p}}=0.92\left(\rho_{\mathrm{s}}=0.08\right)$; the original polymer (Schulz-Zimm (SZ) distribution, $X_{w}{ }^{0} / X_{n}{ }^{0}=2.8, X_{w}{ }^{0}=1810$ ); $p=0.7$; $v_{\mathrm{p}}{ }^{0}=0.94 \mathrm{vol} \%$. The value of $k$ is indicated on line: open mark, experimental data point $\left(v_{\mathrm{p}}{ }^{0}=0.94 \mathrm{vol} \%\right.$, $\left.\rho_{\mathrm{p}}=0.92\right)$.

polymers in the polymer-rich and polymer-lean phases, respectively. Here, the full line corresponds to $\rho_{\mathrm{p}}=0.04 \quad\left(\rho_{\mathrm{s}}=0.96\right)$ and the broken line to $\rho_{\mathrm{p}}=0.92\left(\rho_{\mathrm{s}}=0.08\right)$ and the $k$ values are indicated on the curves. The open mark is an experimental data point for the PS-MCH system $\left(v_{\mathrm{p}}{ }^{0}=0.94 \mathrm{vol} \%\right.$ and $\left.\rho_{\mathrm{p}}=0.92\right)$. From the experimental view point, the $X_{w} / X_{n}$ value of a polymer precipitate with larger $\rho_{\mathrm{p}}$ has a higher accuracy, and this is the case. The effect of the $k$-parameter on $X_{w} / X_{n}$ of the polymer in the two phases becomes significantly large as the average-molecular weight of the original polymer decreases and as $\rho_{\mathbf{p}}$ of the polymer in the polymerrich phase or $\rho_{\mathrm{s}}$ of the polymer in the polymer-lean phase decreases. It should be noted that $X_{w} / X_{n}$ of the polymers in the two phases separated under the same conditions except $X_{w}{ }^{0}$, revealed a minimum at a specific $X_{w}{ }^{0}$ if $k \neq 0$. In previous paper, ${ }^{17,18}$ a significant increase in $X_{w} / X_{n}$ of the polymers obtained in the same manner could be finally observed with $X_{w}{ }^{0}$ in the case of $k=0$. Comparison of the actual experiment with the theoretical calculation leads to the conclusion that for the PS-MCH system, $\left(v_{\mathrm{p}}{ }^{0}=0.94 \mathrm{vol} \%, \rho_{\mathrm{p}}=0.92\right.$ and the phase separation temperature $T_{\mathrm{c}}=20^{\circ} \mathrm{C}$ ) $k$ lies between 3 and 10. From the above discussion, we further conclude that for a low-molecular weight 


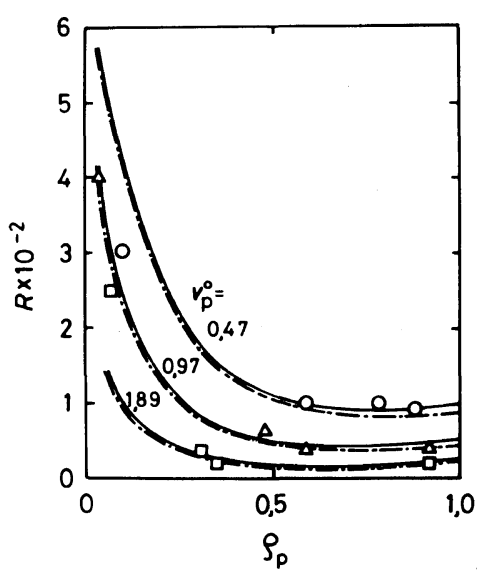

Figure 16. Relations between the volume ratio, $R$, and the fraction size, $\rho_{\mathrm{p}}$ : open circle, experimental data points for polystyrene $\left(M_{w}=23.4 \times 10^{4}, M_{n}=8.9 \times 10^{4}\right)$ in methylcyclohexane; $v_{\mathrm{p}}{ }^{0}=0.47 \mathrm{vol} \%$ (circle), $0.94 \mathrm{vol} \%$ (triangle), and $1.89 \mathrm{vol}^{\%} \%$ (rectangle $^{2}$; full and chain lines, theoretical curves caluculated by assuming $k=0$ and 10 under the same conditions as those in actual experiments $\left(X_{w}{ }^{0}=1810, p=0.7\right) . v_{\mathrm{p}}{ }^{0}$ value is indicated on the curve.

polymer, the fractionation based on the solubility difference is influenced to a fair extent by the molecular weight dependence of the $\chi$-parameter. In the phase separation and fractionation experiments on PS-CH $\left(X_{w}{ }^{0}=2120\right)$ and PS-MCH $\left(X_{w}{ }^{0}=1810\right)$ systems reported in the previous paper, ${ }^{2}$ respectively, the effect of molecular weight dependence of the $\chi$-parameter can be neglected, if $k$ is less than 3 .

For the PS-CH and PS-MCH systems, the volume ratio, $R$, and the polymer volume fraction in the polymer-rich phase, $v_{\mathrm{p}(2)}$, are remarkably influenced by the concentration dependence of the $\chi$ parameter, $p$ (for example, see Figures 13, 14, 24 , and 25 in ref 2). ${ }^{2}$ Contrary to this, the effect of the molecular weight dependence of the $\chi$-parameter is very small as is shown in Figures 16 and 17, where $R$ and $v_{\mathrm{p}(2)}$ are plotted as functions of $\rho_{\mathrm{p}}$. The open, triangle, and rectangle marks are experimental results obtained previously ${ }^{2}$ for PS-MCH system and the full, dotted, broken, and chain lines are the theoretical curve calculated for this system assuming $k=0,1,3$, and 10 , respectively (see Table II). In the previous paper, ${ }^{2}$ we estimated the $p$ value by comparing the experimental $R$ (or $v_{\mathrm{p}(2)}$ ) value with theoretical $R$ (or $v_{\mathrm{p}(2)}$ ) value. The theoretical curves are well within the experimental uncertainty. This

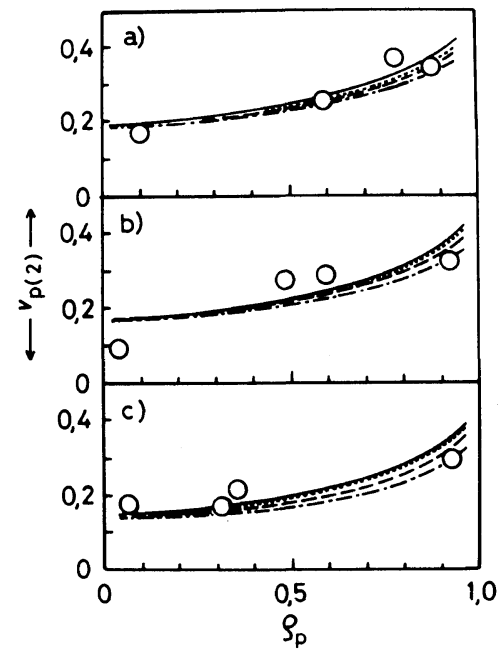

Figure 17. Relations between the polymer-volume fraction of polymer in a polymer-rich phase $v_{\mathrm{p}(2)}$ and the relative amount of the polymer (i.e., the fraction size) $\rho_{\mathrm{p}}$ : open circle, experimental data points for polystyrene $\left(M_{w}=23.2 \times 10^{4}, M_{n}=8.9 \times 10^{4}\right.$ in methylcyclohexane ${ }^{2}$; full, dotted, broken, and chain lines, theoretical curves calculated by assuming $k=0,1,3$, and 10 , under the same conditions as those in actual experiments [the Schulz-Zimm distribution, $X_{w}{ }^{0} / X_{n}{ }^{0}=2.8, X_{w}{ }^{0}=1810$, $p=0.7, v_{\mathrm{p}}{ }^{0}=0.47 \mathrm{vol}^{\circ} \%$ (a), $0.94 \mathrm{vol}^{\circ} \%$ (b), and $1.89 \mathrm{vol} \%$ (c)].

indicates that the method for estimating $p$ employed before has a relatively high reliability, even if the $\chi$ parameter has a molecular weight dependence, and it does not seem possible to estimate the $k$ parameter form $R$ or $v_{\mathrm{p}(2)}$.

From the same data used in Figures 6 and 9 for the PS-MCH system, we plotted $M_{w} / M_{n}$ of the precipitate against the fraction size $\rho_{\mathrm{p}}$ in Figure 18. The simbols have the same meaning as in Figures 16 and 17. As evident from Figure 9, the effect of the molecular weight dependence of the $\chi$-parameter on $M_{w} / M_{n}$ of the precipitate becomes drastically significant in the range $k=3-10$. Scattering of the data point in Figure 17 does not allow an estimation of the $k$ value accurately by a comparison with the theoretical curve. But the $k$-parameter is expected to lie between 0 and 10 (probably, 0 and 6). Clearly the $k$-parameter evaluated from Figure 18 for the polymers with large $\rho_{\mathrm{p}}(\mathrm{ca} .0 .9)$ in the polymer-rich phase is approximately 10 or so, and this is considerably larger than those for the fractions by SPF with small $\rho_{\mathbf{p}}$. This tendency may be closely 


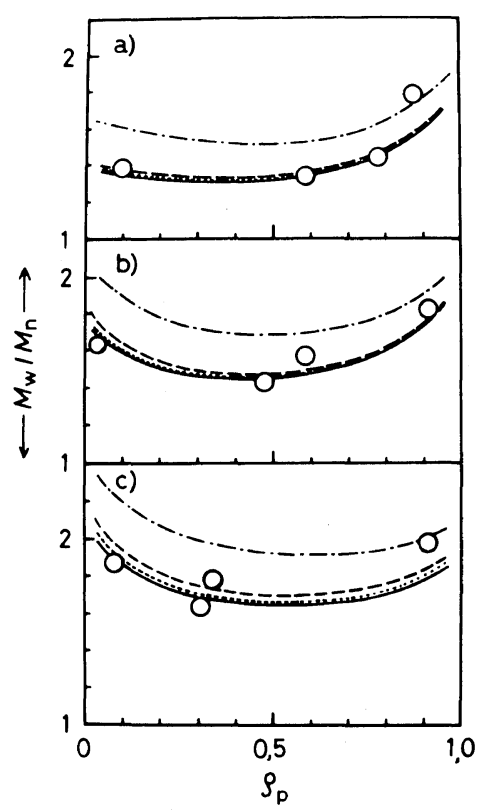

Figure 18. Dependence of the ratio $M_{w} / M_{n}$ of polymer in a polymer-rich phase on the relative amount of the polymer (i.e., the fraction size) $\rho_{\mathrm{p}}$. Open circle and curves have the same meanings as in Figure 17.

associated with the effect that the theory deviates notably from the experiment at larger $\rho_{\mathrm{p}}$. Note that larger $\rho_{\mathrm{p}}$ corresponds to lower phase-separation temperature and that Figure 18 suggests larger $k$ at lower temperatures. This is in good agreement with the results calculated from the partition coefficient (see Figure 4 and Table II).

The general applicability of the conclusions drawn above on the effect of the $k$ - and $p$ parameters on the volume ratio $R$, the polymer volume fraction $v_{\mathrm{p}(2)}$ and $X_{w} / X_{n}$ of the polymers in the two phases was examined by hypothetical phase separations: A $1.0 \mathrm{vol} \%$ solution of the SchulzZimm-type polymer $\left(X_{w}{ }^{0} / X_{n}{ }^{0}=2\right.$ and $\left.X_{w}{ }^{0}=300\right)$ in a single solvent was phase separated to give a relative amount of the polymers in the polymer-rich phase $\rho_{\mathrm{p}}=0.1$ and 0.01 or a relative amount of polymer remaining in the polymer-lean phase $\rho_{\mathrm{s}}=0.1$ and 0.01 , respectively. The results are shown in Figure 19, where the $p$-parameter was changed from 0 to 1.0 at constant $k(=0)$ (broken line) or the $k$-parameter was varied over the range $0-10$ at constant $p(=0)$ (full line). These $p$ and $k$ ranges are those which can be expected in actual polymer-

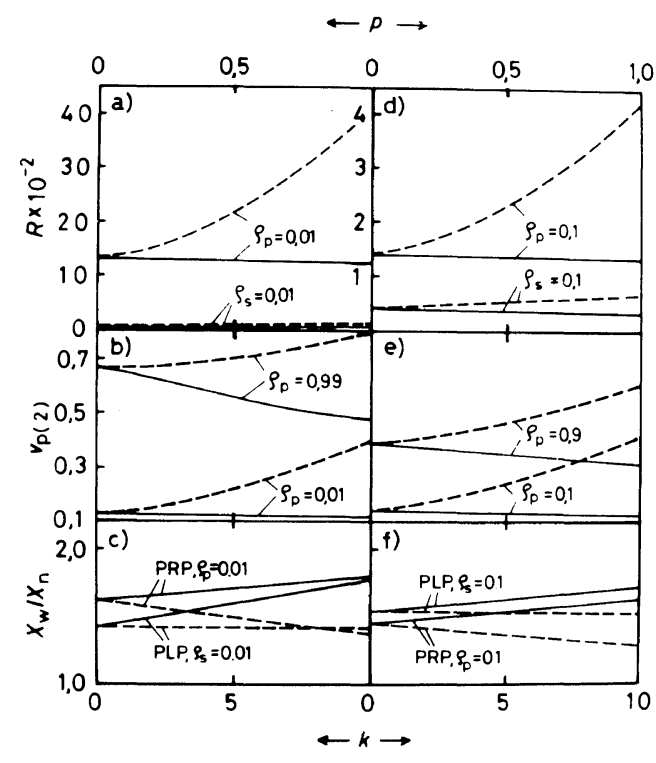

Figure 19. Effect of the $p$ - and $k$-parameters on the polymer volume fraction in a polymer-rich phase $v_{\mathrm{p}(2)}$, the volume ratio of the two phases $R$, and the ratio $X_{w} / X_{n}$ of the polymer in a polymer-rich phase and polymer-lean phase: the original polymer (Schulz-Zimm distribution, $\left.X_{w}{ }^{0} / X_{n}{ }^{0}=2, X_{w}{ }^{0}=300\right)$; a), $\rho_{\mathrm{p}}=0.01$ and 0.99 ; b) and c), $\rho_{\mathrm{p}}=0.01$ and $\left.\rho_{\mathrm{s}}=0.01 ; \mathrm{d}\right), \rho_{\mathrm{p}}=0.1$ and $\left.0.99 ; \mathrm{e}\right)$ and f), $\rho_{\mathrm{p}}=0.1$ and $\rho_{\mathrm{s}}=0.1$; full line, $p=0$ and $k=0-10$; broken line, $k=0$ and $p=0-1.0$.

\section{solvent systems.}

The $R$ is practically independent of $k$-parameter, and in particular, at small $\rho_{\mathrm{p}} R$ is not effected by the $k$-parameter as well as the $p$-parameter. The effect of the $k$-parameter on $v_{\mathrm{p}(2)}$ is negligibly small at small $\rho_{\mathrm{p}}$, but is almost equivalent to the effect of $k$ parameter on $v_{\mathrm{p}(2)}$ at larger $\rho_{\mathrm{p}}$. The ratio $X_{w} / X_{n}$ in a polymer rich phase depends on the $k$ - and $p$-parameters even at small $\rho_{\mathrm{p}}$ or small $\rho_{\mathrm{s}}$, this ratio in polymer-lean phase is $k$-parameter dependent and $p$-parameter independent. Therefore, the $p$ parameter can be accurately estimated from $v_{\mathbf{p}(2)}$ at small $\rho_{\mathrm{p}}$ or from $R$ at relatively larger $\rho_{\mathrm{p}}$, without knowledge of the $k$-parameter. The $k$-parameter has significant effect on $X_{w} / X_{n}$ in a polymer-lean phase at small $\rho_{\mathrm{s}}$.

Figure 20 shows the relation between $M_{w} / M_{n}$ of the fractions isolated by SPF (open mark) or SSF (closed mark) and their $M_{w}$ for PS-MCH system. The circles are experimental data ${ }^{2}$ and the full, dotted, broken, and chain lines are the correspond- 


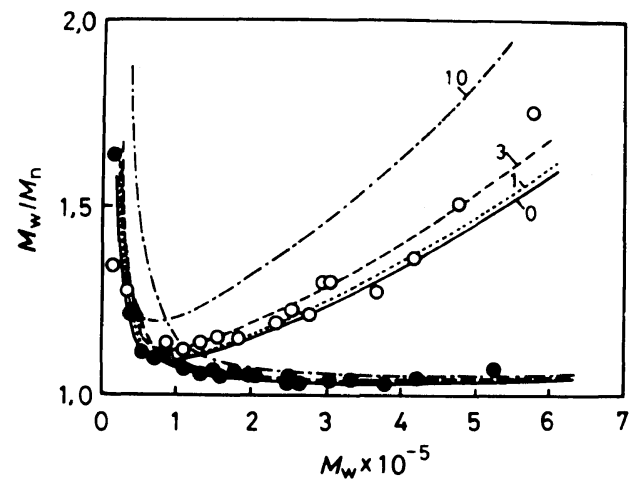

Figure 20. Effect of the $k$-parameter on the relations between the ratio $M_{w} / M_{n}$ and $M_{w}$ of the fractions separated by successive precipitational and successive solutional fractionations (SPF and SSF): circle, experimental data points for polystyrene $\left(M_{w}=23.2 \times 10^{4}\right.$, $M_{n}=8.9 \times 10^{4}$ ) in methylcyclohexane by SPF (open mark) and by SSF (closed mark) ${ }^{2}$ : full, dotted, broken, and chain lines, theoretical curves calculated by assuming $k=0,1,3$, and 10 , under the same conditions as those in actual experiments (Schulz-Zimm distribution, $X_{w} / X_{n}=2.8, X_{w}=1810, p=0.7, v_{\mathrm{p}}{ }^{0}=0.94 \mathrm{vol} \%$. Total number of the fractions in a run, $n_{\mathrm{t}}=18(\mathrm{SPF})$ and 20 (SSF).

Table IV. Comparison of the effect of $k$ - and $p$-parameters on phase-equilibrium characteristics

\begin{tabular}{|c|c|c|c|c|}
\hline $\begin{array}{l}\text { Phase-equilibrium } \\
\text { chracteristics }\end{array}$ & & Comparative role & & Remarks \\
\hline Partition coefficient & & At limit of $\rho_{\mathrm{p}} \rightarrow 0$ & $k<p^{\mathrm{a}}$ & $\begin{array}{l}\text { Figure } 15 \text { of ref } 2, \\
\text { Figure } 2\end{array}$ \\
\hline Volume ratio $R$ & & At limit of $\rho_{\mathrm{p}} \rightarrow 1$ & $\begin{array}{l}k>p^{\mathrm{b}} \\
k \ll p\end{array}$ & $\begin{array}{l}\text { Figures } 14 \text { and } 24 \text { of } \\
\text { ref } 2 \text {, Figure } 16\end{array}$ \\
\hline $\begin{array}{l}\text { Polymer volume fraction } \\
\text { in polymer-rich phase } v_{\mathrm{p}(2)}\end{array}$ & & & $k \ll p$ & $\begin{array}{l}\text { Figures } 13 \text { and } 25 \text { of } \\
\text { ref } 2 \text {, Figure } 17\end{array}$ \\
\hline $\begin{array}{l}\text { Polymer volume fraction } \\
\text { in polymer-lean phase } v_{\mathrm{p}(1)}\end{array}$ & & & $k \approx p$ & \\
\hline \multirow[t]{6}{*}{$M_{w(2)} / M_{n(2)}$} & & At limit of $\rho_{\mathrm{p}} \rightarrow 0$ & $k<p$ & Figure 19 of ref 14 \\
\hline & & $\begin{array}{l}\text { At limit of } \rho_{\mathrm{p}} \rightarrow 0 \\
\text { and large } p\end{array}$ & $k>p$ & \\
\hline & & $\begin{array}{l}\text { At limit of } \rho_{\mathrm{p}} \rightarrow 0 \\
\text { and small } p\end{array}$ & $k<p$ & Figures 11 and 18 \\
\hline & & $\begin{array}{l}\text { At limit of } \rho_{\mathrm{p}} \rightarrow 1 \\
\text { and large } p\end{array}$ & $k>p$ & \\
\hline & SPF & $\begin{array}{l}\text { Initial few } \\
\text { fraction }\end{array}$ & $k<p$ & Figure 21 of ref 2 \\
\hline & & End few fractions & $k>p$ & Figure 20 \\
\hline \multirow[t]{4}{*}{$M_{w(1)} / M_{n(1)}$} & & At limit of $\rho_{\mathrm{s}} \rightarrow 0$ & $k>p$ & \\
\hline & & $\begin{array}{l}\text { At limit of } \rho_{\mathrm{s}} \rightarrow 1 \\
\text { and large } p\end{array}$ & $k>p$ & Figure 12 \\
\hline & & $\begin{array}{l}\text { At limit of } \rho_{\mathrm{s}} \rightarrow 1 \\
\text { and small } p\end{array}$ & $k<p$ & \\
\hline & SSF & $\begin{array}{l}\text { Initial few } \\
\text { fractions }\end{array}$ & $k>p$ & $\begin{array}{l}\text { Figure } 21 \text { of ref } 2, \\
\text { Figure } 20\end{array}$ \\
\hline
\end{tabular}

${ }^{a} p$-parameter is much effective than $k$-parameter.

b $k$-parameter is much effective than $p$-parameter. 
ing theoretical curves calculated by assuming $k=0$, 1,3 , and 10 , respectively. In SPF, the $M_{w} / M_{n} v s . M_{w}$ curve is significantly affected by the $k$-parameter, being larger for larger $k$. The $k$-parameter is estimated approximately to be $1-3$ from comparison of SPF experiment with the theory in Figure 20. It is strongly recommended in SPF to employ a solvent with a $k$-parameter as small as possible. In contrast to this, in SSF, it is apparently unnecessary to consider the effect of $k$-parameter on $M_{w} / M_{n}$ of the fractions, at least except for a few initial fractions.

In the phase separation experiments on PS- $\mathrm{CH}$ and PS-MCH systems, the $k$-parameter influences significantly (a) the molecular weight dependence of the partition coefficient $\sigma$, (b) the MWD of the polymer in a polymer-rich phase or in a polymerlean phase when $\rho_{\mathrm{p}}$ or $\rho_{\mathrm{s}}$ is very small, and (c) the relation between $M_{w} / M_{n}$ of the fractions, isolated by SPF, and their $M_{w}$. Table III summarizes the $k$ values estimated for the above polymer-solvent systems by using method (a)-(c), together with the molecular weight dependence of the $\chi$-parameter. The $k$ value differs depending on the methods employed and temperature, at which the phase separation occurred or at which the osmotic pressure was measured. For example, the value estimated from the coefficient $\sigma$ (a) is about twice as large as from the SPF data (c). Because experimental uncertainty involved in these is least for (c), $k \sim 3$ can be estimated for PS-MCH system. But, there are still questions concerning the true value of the $k$ parameter for this system. A more detailed quantitative discussion of the $k$-parameter, though worthwhile, is beyond the scope of this paper, and left for future study. The relations between $v_{\mathrm{p}}{ }^{0}$ or $R$ and $\rho_{\mathrm{p}}$ are apparently much less influenced by the $k$ parameter than the corresponding relations of $\sigma$, MWD, and $M_{w} / M_{n}$ vs. $M_{n}$.

We conclude that the molecular weight dependence of the $\chi$-parameter has a minor effect, when compared with its concentration dependence, on the phase-separation phenomenon of polymer solutions, but this effect is not always negligible and comes into play with the following characteristics: partition coefficient $\sigma$, MWD of the polymer with small $\rho_{\mathrm{p}}$ in the polymer-rich phase or/and with small $\rho_{\mathrm{s}}$ in the polymer-lean phase, the $M_{w} / M_{n}$ vs. $M_{w}$ relations for the fractions isolated by SPF. It should be noted that at the limit $\rho_{\mathrm{p}} \rightarrow 1$, the polymers remaining in the polymer-lean phase are absolutely independent of the $p$-parameter and in the above regions, the $k$-parameter may play an important role. We can interpret the previously observed small discrepancy between the actual experiments on PS$\mathrm{CH}$ and $\mathrm{PS}-\mathrm{MCH}$ system and the theory, by the molecular weight dependence of the $\chi$-parameter. The fractionation efficiency decreases with an increase in the molecular weight dependence of the $\chi$-parameter.

The effect of the $k$ - and $p$-parameters on the phase-equilibrium characteristics is very complicated, but the comparative role of the two parameters is summerized in Table IV.

Acknowledgement. The authors are grateful to Professor Toru Kawai for his continuous encouragement and stimulating discussions given during the course of this study. We should like to express our gratitude to Dr. Ken Sugamiya of Asahi Chemical Industry Company for his assistance in deriving eq 5 .

\section{REFERENCES}

1. see, for example, K. Kamide, "Fractionation of Synthetic Polymers," L. H. Tung Ed., Marcel Dekker, Inc. New Y,ork, N.Y., 1977, Chapter 2.

2. K. Kamide, Y. Miyazaki, and T. Abe, Makromol. Chem., 177, 485 (1976).

3. K. Kamide, Y. Miyazaki, and T. Abe, Polym. J., 9, 395 (1977).

4. K. Kamide, K. Sugamiya, T. Kawai, and Y. Miyazaki, Polym. J., 12, 67 (1980).

5. R. Koningsveld and L. A. Kleintjens, Macromolecules, 4, 637 (1971).

6. J. W. Kennedy, M. Gordon, and R. Konigsveld, J. Polym. Sci., C, 39, 43 (1972).

7. I. Fujiwara, Ph. D. Dissertation, Osaka Municipal University, 1979.

8. K. Kamide and K. Sugamiya, Makromol. Chem., 139, 197 (1970).

9. K. Kamide, T. Ogawa, M. Sanada, and M. Matsumoto, Kobunshi Kagaku, 25, 440 (1968).

10. Th. G. Scholte, Eur. Polym. J., 6, 1063 (1970).

11. Th. G. Scholte, J. Polym. Sci., A-2, 9, 1553 (1971).

12. W. R. Krigbaum, J. Am. Chem. Soc., 76, 3758 (1954).

13. K. Kamide and K. Sugamiya, Makromol. Chem., 156, 259 (1972).

14. K. Kamide, Y. Miyazaki, and K. Yamaguchi, Makromol. Chem., 173, 157 (1973).

15. K. Kamide and Y. Miyazaki, Makromol. Chem., 176, 1447 (1975).

16. K. Kamide and C. Nakayama, Makromol. Chem., 


\section{Effect of MW Dependence of $\chi$ on Phase Equilibrium}

129, 289 (1969).

17. K. Kamide and Y. Miyazaki, and K. Sugamiya, Makromol. Chem., 173, 113 (1973).
18. K. Kamide and Y. Miyazaki, Makromol. Chem., 176, 1029 (1975). 